\title{
Aprender a Convivir en Educación Superior desde la Práctica Docente, para una Sociedad Democrática
}

\author{
Alma D. Torres-Rivera* y Rodrigo Florencio da Silva \\ Escuela Superior de Comercio y Administración - Unidad Santo Tomás del Instituto Politécnico Nacional, \\ Sección de Estudios y Posgrado, Prolongación de Carpio 471, Colonia Plutarco Elías Calles, Delegación \\ Miguel Hidalgo, C.P. 11340, México, Ciudad de México. (e-mail: atorresri@ipn.mx; rflorencio@ipn.mx) \\ * Autor a quien debe ser dirigida la correspondencia
}

Recibido May. 18, 2018; Aceptado Jul. 27, 2018; Versión final Sep. 28, 2018, Publicado Abr. 2019

\begin{abstract}
Resumen
Este estudio fue diseñado como un paso inicial para determinar las estrategias didácticas en el desarrollo de conductas de convivencia con perspectiva de género, desde un enfoque cualitativo que examina las estrategias descritas por los docentes en su planeación. La discusión se genera entonces a partir de los debates teóricos existentes en torno a tres supuestos: 1) las instituciones de educación superior tienen la responsabilidad de comprender el desarrollo de la convivencia desde sus raíces; 2 ) los estudiantes enfatizan niveles de convivencia y construyen otro tipo de relaciones; 3 ) el modelo educativo de las instituciones de educación superior demanda integrar la perspectiva de género. Los resultados de la investigación ofrecen una aproximación a las estrategias didácticas que emplean los docentes y revelan que los profesores inciden en las conductas de convivencia al reelaborar el discurso en el entramado interaccional y relacional. Este es un elemento clave en el desarrollo de capacidades y habilidades que podrían promover una sociedad democrática.
\end{abstract}

Palabras clave: saber convivir; democracia; práctica docente; educación superior

\section{Learning to Live in Higher Education from the Teaching Practice for a Democratic Society}

\begin{abstract}
This study was designed as an initial step to determine the didactic strategies in the development of behaviors of coexistence with a gender perspective, from a qualitative approach that examines the strategies described by teachers in their class planning. The discussion is then generated from existing theoretical debates around three assumptions: 1) higher education institutions have the responsibility of understanding the development of coexistence and its roots; 2 ) students emphasize levels of coexistence and build other types of relationships; 3 ) the educational model of higher education institutions demands integrating the gender perspective. The results of the research offer an approximation to the didactic strategies used by teachers and reveals that they have an impact on the behaviors of coexistence and rework the discourse in the interactional and relational framework. This is a key element in the development of skills and abilities that could promote a democratic society.
\end{abstract}

Keywords: know how to live; democracy; teaching practice; higher education 


\section{INTRODUCCIÓN}

La educación superior es entendida como el tramo de formación escolar en el que interactúan dos categorías exclusivas: los que enseñan e investigan y los que acuden para ser formados como profesionales. Bajo esta óptica, se promueve el aprendizaje a lo largo de la vida ante la demanda de garantizar la pertinencia y relevancia social de la formación profesional de los egresados en la construcción de una sociedad democrática (Bicocca, 2014; Fernández, 2008). En este contexto, cuando se observan las instituciones de educación superior analíticamente con un alto nivel de desagregación, la atención se orienta a las etapas u operaciones de los procesos académicos, administrativos, sociales y políticos que en ella ocurren, entonces se develan las relaciones lógicas entre las diferentes figuras del hecho educativo. Por tanto, entender, diseñar y ejecutar los procesos de gestión educativa de la institución y su relación con el entorno, requiere de establecer la arquitectura de las acciones de convivencia, como eje estratégico que dinamiza la participación de la comunidad para abrir espacios de coexistencia que gradualmente transforman la organización escolar, el papel del docente y su relación con los diferentes sectores sociales, en torno a convertir el proceso de enseñanza-aprendizaje en un espacio de convivencia y ciudadanía (Domínguez, et al. 2017).

La convivencia en la construcción de una sociedad democrática, se asume como marco discursivo de la reforma educativa de las instituciones de educación superior y del papel de los docentes (Jares, 2002), ya que muchos de los problemas en la construcción de los espacios de convivencia se centran en cómo los estudiantes manejan el conflicto en las relaciones alumno-profesor, relación entre iguales, la violencia escolar (intimidación con amenazas, agresiones verbales, agresiones físicas, aislamiento social o discriminación). Por ejemplo, Castro, et al. (2014) han encontrado un alto nivel de prevalencia de violencia entre los estudiantes de las escuelas de negocios, más que en otras unidades académicas, estos estudios generaron un sentimiento de que la educación de nivel superior no está respondiendo adecuadamente en términos de las competencias de convivencia dentro de la institución y en la sociedad. La convivencia debe ser uno de los pilares educativos del siglo XXI y la escuela es un entorno en el que las interacciones tienen lugar, donde los alumnos pasan una gran cantidad de tiempo y es un entorno social adecuado para aprender a convivir (García-Raga, 2017).

Hess y Colli (2018) mencionan que el currículo en la educación está diseñado para asegurar que los estudiantes tengan las habilidades necesarias para aprender a un nivel riguroso con una amplia perspectiva en una variedad de temas contemporáneos, como parte de una educación que al mismo tiempo les proporciona las bases para su formación profesional. La exigencia al currículo en la educación de nivel superior es preparar para la convivencia a los egresados en los entornos del ejercicio profesional. No obstante, que un gran número de docentes no están entrenados para enseñar la convivencia, solo se remite a brindar información que se limita a la resolución de los problemas prácticos o al ejercicio racional de su conducta. En este sentido, existe un gran vacío en las reformas educativas que enfatice la formación para establecer una relación entre los estudiantes. A lo largo de este debate, irónicamente existe un énfasis en incrementar la enseñanza de la convivencia, el reconocimiento de las diferencias culturales, así como de las prácticas de responsabilidad social y sustentabilidad, ya que éstas tienen una implicación significativa en la construcción del bien común social desde el ejercicio de la profesión. La comunidad científica reconoce que la gestión de la convivencia en las escuelas es un complejo multidimensional en el marco de un proceso global que integra otros factores como la gestión de recursos, la programación y otras variables favorecen un clima positivo de convivencia (lanni, 2003; Vélez et al. 2017).

Mejorar la convivencia al promover relaciones basadas en el respeto y la aceptación de los demás, desde las escuelas a través de programas educativos que desarrollen en los estudiantes comportamientos comprensivos, críticos y actitudes asertivas para adquirir una conciencia analítica que les permita comprender el mundo que los rodea y sus posibles cambios (Tuvilla, 2004 citado por Pérez et al., 2016). Para Celkan et al., (2015), cuanto mayor sea el nivel de comunicación con el docente, mejor las relaciones entre los actores del hecho educativo y cuando existen buenas relaciones entre los estudiantes y los profesores, el respeto indudablemente sería mutuo. Como parte de sus tareas, en las instituciones de educación de nivel superior, la problemática de la convivencia se está debatiendo de forma colegiada más seguido, ya que los esfuerzos de los docentes se asumen con carácter formativo tanto por los conocimientos técnico-especializados, necesarios para el desarrollo extraordinario de una profesión como por una formación que cultive la humanidad (Nussbaum, 2010). Sin embargo, raramente, se reconoce que las estrategias didácticas diseñadas por los docentes tengan la intención de promover la convivencia en los estudiantes.

Este tipo de esfuerzos del docente puede consistir en lograr la simetría relacional y comunicativa entre docentes y estudiantes, a través de una cultura institucional que refuerce la democracia. Aquí el problema de la convivencia como entramado interaccional y relacional, será clave en el desarrollo capacidades que podrían promover una democracia. Knowles y Clark (2018) mencionan que los docentes tienen iniciativas que toman en serio los defectos de la democracia y examinen las nociones de educación democrática para reflejar con mayor precisión las realidades sociales. En este contexto, el cuestionamiento de cómo las escuelas 
implementan un programa de convivencia en los escenarios escolares de nivel superior. Frente a ello, los docentes desde su práctica en el aula favorecen el desarrollo de trabajo colaborativo y cooperativo a partir de la integración de equipos de trabajo, dentro del marco de referencia del modelo educativo que promueve el desarrollo en los estudiantes de conductas de tolerancia y coexistencia.

En esta investigación se aborda la convivencia desde una perspectiva cualitativa, con el desarrollo de las estrategias didácticas descritas por los docentes. También se explora cómo la institución (explícita o implícitamente) contribuye a la convivencia. Apuntamos a presentar un modelo contemporáneo de la relación entre las estrategias didácticas en el desarrollo de comportamientos respetuosos, de dialogo, elocuencia y asertividad que utilizan los docentes. El estudio fue diseñado como un paso inicial en la asistencia para las instituciones de educación para determinar las estrategias didácticas en el desarrollo de conductas de convivencia con perspectiva de género. Iniciamos con tres supuestos: (1) las instituciones de educación superior tienen la responsabilidad de comprender el desarrollo de la convivencia y sus raíces (Kim, 2016; GutiérrezMéndez y Pérez-Archundia, 2015 Castro, et al., 2014) (2) la población de estudiantes está cambiando y va mostrando el crecimiento de los niveles de tolerancia formados desde una dimensión convivencia en la construcción de las relaciones con otros y para otros (Medina, 2016); y (3) El modelo educativo de las instituciones de educación superior demanda el incremento de los niveles de convivencia a partir de reconocer los niveles de violencia escolar (Ianni, 2003). Por y a lo largo de la influencia en la convivencia de los estudiantes a nivel superior, las escuelas han tenido un efecto profundo desde la perspectiva de género. Hay preocupación de que la evolución de los cambios en las escuelas mueva tanto el currículo como a los actores institucionales fuera de la formación para la vida. La preocupación por la mejora cualitativa de la educación está presente en la práctica totalidad de los países desarrollados o en vías de desarrollo (Ferrer, 2008). Pocas instituciones se encuentran analizando el papel de la figura del profesor y la importancia de la convivencia, así como de la compresión del modelo educativo en la formación de un ciudadano para vivir en una sociedad democrática.

\section{OTROS ANTECEDENTES}

Vivimos en una sociedad que experimenta una profunda crisis de valores, donde la desigualdad social aumenta y la coexistencia es cada vez más difícil (Gutmann, 2001). El debate teórico se centra en el papel protagónico de las instituciones de educación superior en comprender el desarrollo de la convivencia y sus raíces, ya que la exclusión social trasciende la pobreza y tiene relación con la falta de participación en la sociedad. sin acceso a los bienes básicos de bienestar social (Unesco, 2008). El sistema educativo es una plataforma que tiene la finalidad de alcanzar, por diferentes medios equivalentes en aprendizajes comparables para todos y con calidad (Flores y Lavín, 2017), esto significa integrar la perspectiva de género, como una construcción social dentro de los contextos históricos y cultural, que se relacionan con los roles sociales de los individuos que son dados o forzados por la sociedad (Cuesta y Witt, 2014). Horbath y Gracia (2016) dicen que la educación tiene la necesidad de formar a los estudiantes para la convivencia demandando la generación de bases y aprendizaje para una convivencia sana, por lo tanto el docente es un agente de transformación al implementar estrategias didácticas que rescaten la igualdad entre los géneros, que propicien la tolerancia y contribuyan a prevenir la violencia y el conflicto para asegurar el desarrollo integral de las personas, en particular en los contextos de crisis, desde dos tipos de ejes: los estudiantes enfatizan niveles de convivencia y construyen otro tipo de relaciones y el modelo educativo integran la perspectiva de género.

Aprender a convivir dentro y fuera del aula, es una construcción social que se enseña en el seno de las instituciones a partir de una serie de compromisos que se asumen en lo individual y se ejercen en lo colectivo, con el reconocimiento de que aprender a vivir juntos es la base de autoafirmación personal. Esta autoafirmación y la forma de interrelacionarse con sus pares, como componente de la convivencia requiere de aprender a comunicarse con asertividad, es a través de la comunicación que se dialoga y se expresan mensajes en igualdad de condición e interacción a partir de seguir las reglas, reconocer la diversidad de las personas, al escuchar, dialogar, reflexionar y disentir sin romper la convivencia al aceptar que somos parte de un todo. La convivencia pauta las acciones de interrelación entre estudiantes y docentes al formase como ciudadanas y ciudadanos comprometidos, críticos y con conciencia de la época que les toca vivir. Martínez (2011) menciona que en el aula el docente se vuelve responsable de la formación de las jóvenes generaciones, es así que el desafío en el proceso de enseñanza aprendizaje es impulsar procesos de democratización.

Por lo tanto, la convivencia es un punto fundamental en la formación del futuro profesional que se está formado en las instituciones de educación superior para el ejercicio de la ciudadanía. En este sentido, en el diseño de estrategias didácticas el docente está interesado en una aprendizaje que permita desarrollar las habilidades para relacionarse con otros individuos, actuar y participar de manera responsable en las decisiones colectivas, por lo tanto su puestas en marcha dentro del aula, implica que el docente busque desarrollar la capacidad que tiene el estudiante para entender, valorar y aceptar la diversidad desde los puntos de vista de otros, y fomentar la participación tanto en los espacios de lo escolar como en el ejercicio de la profesión. En otras palabras, la ciudadanía tiene como característica que las personas viven en sociedad y le dan sentido a su actuación. 
Las escuelas de educación superior, tienen presente en la construcción de un currículo flexible y significativo que las estrategias didácticas son pertinentes en la medida que desarrollan los saberes de convivencia que exige el ejercicio de un profesional, es así que las actividades que plantea en su secuencia didáctica tienen un papel vital en el desarrollo de una cultura de la convivencia. Los docentes de educación superior implican en su práctica en el aula los saberes disciplinarios, pedagógicos, de la realidad sociopolítica y económica del país y el saber ser como persona que asume lo ético y mediador (Lodoño, 2010). La preocupación del académico por una formación integral, por una enseñanza pertinente con el ejercicio de una profesión en favor del desarrollo personal y profesional del estudiante que fomentan el mejoramiento y reconocimiento de los comportamientos que fortalecen las acciones para la construcción, integración e interacción con tolerancia, autonomía y creatividad en la resolución del conflicto en los contextos escolares pauta espacios más democráticos (Torres, et al., 2014). Bandura y Menlove (1968) señalan que los estudiantes aprenden juntos, de allí que el clima del aula promueva relaciones interpersonales a partir de actitudes y valores que van modelando la personalidad a través de las experiencias vividas que se configuran en el proceso de aprendizaje. Aprender a convivir es constitutivos de la democrática. Es decir, el aula es el espacio de intercambio, vinculación, reciprocidad, escucha y comunicación, participación, compromiso, consenso y reflexión. Condiciones que se dan a través de la práctica docentes y se hacen explicitas en los proyectos escolares que atienden a demandas tanto curriculares, institucionales y sociales en función de alcanzar las metas propuestas de responsabilidad y de pertenencia (Ianni, 2003).

El docente favorece el proceso de aprendizaje en el aula cuando al pensar los métodos, técnicas y procedimientos tiene como finalidad enseñar estratégicamente los contenidos curriculares en el contexto del ejercicio de una profesión (Díaz, 2009). Vygotsky (1979) señala que las estructuras y procesos mentales están establecidas en las interacciones con los demás, el aprendizaje significativo se promueve con la reflexión pedagógica de los docentes sobre el aprendizaje de convivir a través de la socialización de los estudiantes en situaciones de interrelaciones que favorezca el desarrollo social, lo que pauta las estrategias docentes que se utilizan para aprender a ser y a convivir en sociedad, asumiendo que el estudiante está implicado activamente en la construcción del conocimiento, es decir que el aula se transforma en un espacio de comunidades de aprendizaje que abordan los conflictos en el ejercicio de la profesión con sus habilidades de diálogo, negoción y relacionales.

Una herramienta útil para lograr un aprendizaje significativo es el desarrollo de la planeación didáctica, ya que en este proceso se seleccionan conjuntos actividades diseñadas (secuencia didáctica) por el docente para promover el aprendizaje significado de los saberes conceptuales, procedimentales y actitudinales que articulan la relación docente-contenido-realidad-estudiante (Díaz, 2009). Desde el enfoque constructivista, la relación docente-contenido-realidad y estudiante, hace indispensable trabajar los valores sociales que forman el individuo. La convivencia es considerada un valor que debe ser tratada a lo largo de la vida (Díaz y Hernández, 2002; lanni, 2001; Coll et al., 1999; Rivière, 1987), subyace fomentar el proceso de individualización y de socialización, en construcción de una identidad enmarcada por un entorno cultural y social. Esto compromete en la función del docente despliegue un proceso de organización, selección y transformación de las relaciones entre los saberes conceptuales, procedimentales y actitudinales que se integran en el desempeño efectivo del ejercicio profesional. Con respecto a los saberes actitudinales, el currículo favorece que los individuos se guían por el beneficio igualitario, al desarrollo armónico y a la convivencia con democracia y justicia (Martínez y Prats, 2014).

La convivencia es una tarea asignada a la educación que se ofrece en todos los niveles según el documento de la UNESCO (2000) en el foro Mundial de Educación de Dakar, ya que es necesario implementar estrategias didácticas que rescaten la igualdad entre los géneros, con programas educativos que propicien la tolerancia y contribuyan a prevenir la violencia y el conflicto con la finalidad de asegurar el desarrollo integral de las personas, particular en los contextos de crisis. La educación tiene que promover la movilización de los saberes para ser y convivir desde la justicia, la paz y la cooperación, que se asume desde el modelo socio cognitivo (Vygotsky, 1979), siendo la escuela un espacio de convivencia como marco de relaciones personales que son interiorizados en el desarrollo de habilidades para convivir, el profesor ejerce un papel estratégico en el desarrollo cognitivo. Fomentar la convivencia demanda del docente el planteamiento de estrategias didácticas pertinentes al estudiante que se formalicen a partir de propósitos concretos establecidos de forma transversal en cada uno de los programas de estudio que integran el plan de formación profesional que posibiliten el vivir juntos entre iguales reconociendo la diversidad sin violencia y tolerancia. Ciertas formas metodológicas y técnicas didácticas que demostraron ser eficientes para utilizar directamente con los saberes comportamentales son las técnicas participativas (dinámicas y socio-dramas), involucrando a los estudiantes en las decisiones (Coll, et al., 1999). Dado lo expuesto, la pregunta de investigación que guía el estudio es ¿cuáles son las estrategias didácticas que implementan los docentes de educación superior para fomentar la convivencia desde la perspectiva de género en el programa académico de contador público de la Escuela Superior de Comercio y Administración del Instituto Politécnico Nacional? 


\section{METODOLOGIA}

La presente investigación de tipo exploratoria con enfoque cualitativo, se realizó en la Escuela Superior de Comercio y Administración Santo Tomás del Instituto Politécnico Nacional, dentro de una epistemología constructiva, donde el significado se construye colectivamente dentro de un contexto institucional. En un entorno donde los estudiantes interactúan entre sí sobre cuestiones económicas y administrativas de las empresas que son tratadas desde lo contable. Por lo tanto, están dispuestos a discutir los problemas de las empresas y explorar las perspectivas de crecimiento y competitividad, mediante la formulación de una investigación desde el aula y la intervención en la empresa para diseñar una alternativa de solución.

\section{Recolección de datos y codificación}

La Escuela Superior de Comercio y Administración Santo Tomás pertenece al Institutito Politécnico Nacional, elemento contextual que en el marco del modelo educativo establece que el diseño de la planeación didáctica tipo de las unidades de aprendizaje con el trabajo colaborativo de los docentes en el pleno de las academias. La academia de Humanidades diseña la planeación didáctica tipo de la unidad de aprendizaje de Seminario de Investigación Supervisada mediante trabajo colegiado, misma mecánica que sigue la academia de Administración en el diseño de la planeación didáctica de Dirección Estratégica, así como Plan de Negocios. La evidencia de aprendizaje que presentan los estudiantes es el informe de investigación para atender la problemática de una empresa mediante la propuesta de una alternativa de solución, que desarrollan bajo la supervisión técnica y metodológicamente de los docentes. La presentación del informe es la etapa final de la formación profesional. Así que mediante, el estudio de caso como herramienta metodológica desde la teoría constructivista, como fuente de información relevante se seleccionaron seis planeaciones didácticas (véase tabla 1) que después de la evaluación diagnóstico que realizaron al inicio de semestre los docentes que impartieron las asignaturas en el semestre 2015-2016 ajustaron a las características del grupo.

Tabla 1: Distribución de las planeaciones didácticas

\begin{tabular}{llcc}
\hline \multicolumn{1}{c}{ Unidad de aprendizaje } & \multicolumn{1}{c}{ Academia } & Número de docentes & $\begin{array}{c}\text { Número de } \\
\text { planeaciones }\end{array}$ \\
\hline Dirección Estratégica & Negocios & 2 & 2 \\
Plan de Negocios & Finanzas & 3 & 2 \\
Seminario de Investigación Supervisada & Humanística & 3 & 2 \\
\hline
\end{tabular}

La Escuela Superior de Comercio y Administración Santo Tomás tiene el compromiso, de acuerdo con el modelo educativo, de formar Contadores Públicos establece en su mapa curricular unidades de aprendizaje de integración que proporciona a los futuros profesionales herramientas efectivas que apoyen a su inserción en una economía cada vez con menos fronteras que poseen habilidades de negociación, administrativas, de manejo de grupos, liderazgo que puede ajustarse a los diferentes individuos y situaciones, cultural general, creatividad, cooperación y habilidad de trabajo en grupo.

\section{Codificación y análisis}

La colección de datos, a través del estudio cualitativo, se realizó a partir de las categorías que permite identificar en la planeación didáctica, el conjunto de actividades que planea, ejecuta y evalúa el docente, resultado del trabajo colegiado y avalado por las academias. Posteriormente se utilizó el programa de estudios para determinar la consonancia entre la secuencia didáctica, las estrategias y las competencias esperadas en el estudiante, así como los saberes no abordados, hasta alcanzar la saturación de información esperada. Este proceso fue interactivo. Las categorías de análisis fueron establecidas de acuerdo con los componentes generales de la literatura revisada de la convivencia (véase tabla 2 y 3 ). Esto fue derivado de la literatura del modelo a seguir por los actores del proceso de aprendizaje que impacta el desarrollo de la tolerancia en los estudiantes. Sin embargo, tratando de precisar con más detalle quienes estaban involucrados, en qué contexto y cómo era el proceso que los docentes seguían se analizaron las estrategias de cooperación, compromiso y el bien común por su impacto en los valores que promueven la tolerancia. Nuevamente se buscó los aspectos relacionados con la tolerancia y al mismo tiempo se cuidó que no se trastocara la naturaleza orientada al descubrimiento de la búsqueda cualitativa.

\section{Categorías}

Aprende a convivir. - Significa ser capaz de participar y promover interacción con los demás a partir de la comunicación y correspondencia, cooperación, respeto y pertinencia (véase tabla 2), compromiso con el bien común y el cumplimiento de las normas con un sentido de identidad profesional y pertenencia institucional (Fierro-Evans, et al., 2013; lanni, 2003). Después, la discusión en los debates teóricos se centró en el papel 
de las instituciones de educación superior y su responsabilidad para comprender el desarrollo de la convivencia y sus raíces (Medina, et al., 2016), en combinación con el análisis de contenidos de la planeación didáctica se establecieron dos tipos de ejes: los estudiantes enfatizan niveles de convivencia y construyen otro tipo de relaciones y en el modelo educativo se integran la perspectiva de género. Se identificaron las actividades específicas de los estudiantes, consideradas importantes para su desarrollo como la responsabilidad social y la democracia. El reforzamiento de la convivencia en el aula por parte del docente (Jares, 2002) revela que los estudiantes integran la tolerancia mediante la combinación de: a) desarrollo de las oportunidades de empleabilidad en el ejercicio de la profesión, b) el prestigio de la institución, c) código de conducta que está centrado en escuchar a otros.

Tabla 2: Aprender a convivir

\begin{tabular}{|c|c|c|}
\hline Categoría: & \multicolumn{2}{|l|}{ Aprender a convivir } \\
\hline Subcategoría & Descripción & Técnicas \\
\hline $\begin{array}{l}\text { Participación y } \\
\text { promoción de } \\
\text { interacción con los } \\
\text { demás }\end{array}$ & $\begin{array}{l}\text { Crea un ambiente donde se amplían y consolidan lazos } \\
\text { de compañerismo y amistad ejercitando la aceptación, } \\
\text { compresión. }\end{array}$ & $\begin{array}{l}\text { Participación en juego de roles y } \\
\text { socio-dramas } \\
\text { Debate } \\
\text { Expone y explica con carácter }\end{array}$ \\
\hline $\begin{array}{l}\text { Comunicación y } \\
\text { correspondencia }\end{array}$ & $\begin{array}{l}\text { Dialoga abiertamente y pone en práctica los valores } \\
\text { como la confianza, la aceptación de las diferencias. } \\
\text { Actúa en beneficio de todos de acuerdo con sus } \\
\text { derechos, con su condición y con sus circunstancias. }\end{array}$ & $\begin{array}{l}\text { persuasivo } \\
\text { Toma de decisiones }\end{array}$ \\
\hline Cooperación & $\begin{array}{l}\text { Actúa en beneficio de todos de acuerdo con sus } \\
\text { derechos, con su condición y con sus circunstancias. }\end{array}$ & \\
\hline Respeto y pertinencia & $\begin{array}{l}\text { Reconoce el valor inherente de los derechos humanos y } \\
\text { la dignidad del otro y de sí mismo, así como del entorno } \\
\text { y del mundo natural que lo rodea. }\end{array}$ & \\
\hline
\end{tabular}

Estrategia docente. - Conjuntos actividades seleccionadas y diseñadas (secuencia didáctica) por el docente que promueven aprendizajes del significado de saberes actitudinales, que articulan la relación docentecontenido-realidad-estudiante, como la socialización y construcción de una identidad enmarcada por un contexto social y cultural (Domínguez, et al. 2017; Gutiérrez-Méndez y Pérez-Archundia, 2015).

Tabla 3: Estrategia docente (Adaptado de la Guía 49, del Ministerio de Educación Nacional de Colombia, 2013)

\begin{tabular}{|c|c|c|}
\hline Categoría: & \multicolumn{2}{|c|}{ Estrategia docente } \\
\hline Subcategoría & Descripción & Secuencia didáctica \\
\hline Oportunidad para innovar & $\begin{array}{l}\text { Son ideas que pone en práctica el } \\
\text { docente para construir acciones que } \\
\text { benefician el saber convivir. }\end{array}$ & $\begin{array}{l}\text { Definir y reconocer los contextos de } \\
\text { convivencia que preocupan, inquietan o } \\
\text { impactan a la comunidad. }\end{array}$ \\
\hline Lugar de convivencia & $\begin{array}{l}\text { Resalta escenarios del ámbito del } \\
\text { desempeño profesional, donde se } \\
\text { articular las acciones de convivencia }\end{array}$ & $\begin{array}{l}\text { Identificar las situaciones que la comunidad } \\
\text { educativa reconoce que perjudica la } \\
\text { convivencia escolar, se debe hacer un } \\
\text { proceso de priorización de la situación o tema. }\end{array}$ \\
\hline Pregunta frecuente & $\begin{array}{l}\text { Plantea dudas recurrentes y sus } \\
\text { actividades con respecto a la convivencia }\end{array}$ & $\begin{array}{l}\text { Actividades didácticas, a partir de la reflexión } \\
\text { que evidencia situaciones que inciden sobre } \\
\text { la convivencia tanto en el ámbito escolar como } \\
\text { en el desempeño profesional. }\end{array}$ \\
\hline Rincón legal & $\begin{array}{l}\text { Fundamente la formación profesional en } \\
\text { el saber convivir a partir de la } \\
\text { normatividad que aplica en los diferentes } \\
\text { espacios de la escuela y el campo laboral. }\end{array}$ & $\begin{array}{l}\text { Se asegura de que el estudiante entienda la } \\
\text { convivencia y que pueda manejar la } \\
\text { normatividad que aplica en los diferentes } \\
\text { espacios de actuación. }\end{array}$ \\
\hline ¿Sabías que...? & $\begin{array}{l}\text { Plantea datos relevantes, opiniones y } \\
\text { percepciones de la comunidad frente a los } \\
\text { aspectos clave de la convivencia }\end{array}$ & $\begin{array}{l}\text { Proporciona información precisa de los } \\
\text { indicadores conductuales que inciden las } \\
\text { relaciones con otros. }\end{array}$ \\
\hline Recomendaciones & $\begin{array}{l}\text { Se refiere a ideas, propuestas } \\
\text { sugerencias. }\end{array}$ & $\begin{array}{l}\text { Establece recomendaciones sobre lo que } \\
\text { debería hacer la escuela para promover la } \\
\text { convivencia. }\end{array}$ \\
\hline Ejemplo & Presenta casos reales. & $\begin{array}{l}\text { Analiza la situación de mujeres y hombres en } \\
\text { la escuela, haciendo énfasis en el contenido } \\
\text { de convivencia y paz en términos de la } \\
\text { participación, responsabilidad democrática, } \\
\text { pluralidad, identidad y valoración de las } \\
\text { diferencias y calidad de las relaciones en } \\
\text { contexto del aula o el ejercicio de la profesión }\end{array}$ \\
\hline
\end{tabular}


En la tabla 3 se muestra, las subcategorías establecidas a partir de la organización cognitiva de aprendizaje de la convivencia que está asociada al modelo educativo institucional.

\section{Validación}

A partir de la revisión de la literatura se seleccionaron las estrategias docentes para aprendizajes significativos de la convivencia: las técnicas participativas (juego de roles y los socio-dramas), las discusiones y técnicas de estudio activo, el debate, las exposiciones y explicaciones de carácter persuasivo, toma de decisiones mediante el análisis de dos categorías: aprender a convivir y estrategias docentes (Díaz, 2009). Aprender a convivir con seis subcategorías: participación que promueven interacción con los demás, comunicación y correspondencia, cooperación, respeto y pertinencia, compromiso con el bien común, y cumplimiento de las normas; y la categoría estrategia docente compuesta por siete subcategorías oportunidad para innovar, lugar de convivencia, pregunta frecuente: lo que sé, lo que quiero saber, lo que aprendí, cuadro sinóptico y el uso de analogía para tratar los aspectos legales, ¿Sabías que...? como mecanismos para establecer ejemplificaciones y recomendaciones sometidas a expertos para evaluar su validez de contenido.

En el análisis de la información se recurrió al método de comparación constante propuesto en la teoría fundamentada (Glaser y Strauss, 2017), por considerar que las estrategias didácticas que ponen en práctica los profesores en el aula y los diferentes ámbitos del desempeño. En este método se codifican y analizan los datos, interrelacionándolos e integrándolos de forma coherente y de acuerdo a la teoría existente. Con base en Glasser y Strauss (2017) definen categorías para determinar los contenidos dominantes de naturaleza recursiva durante la codificación y búsqueda de temas subyacentes. Las actividades de la secuencia didáctica se consideraron unidades de significado y se organizan en agrupaciones de acuerdo con las categorías de la tabla 2 y 3 dentro del aula. Aparecieron nuevas categorías, ya que algunas de las categorías originales se combinaron o eliminaron. La validez del estudio se asegura con la recolección de las construcciones que se derivan de la secuencia de actividades que realiza el docente en la planeación didáctica, mientras que la confiabilidad de los hallazgos se apoya de las técnicas de codificación y análisis del método constantes. Los límites de este estudio están definidos por el proceso de la planeación didáctica y las estrategias didácticas de aprender a convivir dentro de un marco temporal. Los resultados del estudio no son generalizables ya que responden sólo a la dinámica de un ambiente de aprendizaje en un contexto particular. Sin embargo, la aproximación al aprendizaje de la convivencia como fenómeno de estudio desde la práctica docente en el nivel de educación superior, resulta útil para dar una visión general de la relación entre la práctica áulica, la planeación didáctica y el modelo educativo.

\section{HALLAZGOS}

Los siete factores que describen como el docente integra el saber convivir en su práctica en el aula y desarrolla sus propias estrategias son: oportunidad para innovar, lugar, pregunta frecuente, rincón legal, ¿Sabías que...? y recomendaciones en función de la convivencia. Además, las estrategias docentes son utilizadas para promover en los estudiantes los principios de la democracia y formar sus expectativas de la convivencia con los demás. Dentro del proceso del desarrollo, las estrategias didácticas que plantea el docente contribuyen directamente al conjunto de principios democráticos que definen la participación en la toma de decisiones como marco de referencia del saber convivir. Cuatro de los siete factores que son oportunidad para innovar, lugar o contexto, adaptación al medio y analogías para tratar los aspectos legales, permite registrar que los docentes reconocen la importancia de la convivencia como el dialogo abierto y poner en práctica los valores como la confianza, la aceptación de las diferencias, actuación en beneficio de todos de acuerdo con sus derechos, con su condición y circunstancia, éste es el producto de relaciones que catalizan la democracia, permitiendo así una formación integral de los estudiantes. En suma, una relación interactiva que reconoce el valor inherente de los derechos humanos y la dignidad del otro y de sí mismo, así como del entorno y del mundo natural que lo rodea. Este proceso ayuda a determinar la evolución de la trayectoria del desarrollo del saber convivir (el grado de tolerancia, toma de decisiones y perspectiva de género). El reforzamiento de la convivencia en el aula presente en las estrategias didácticas retrata el desarrollo del saber convivir como un proceso holístico anclado al manejo institucional con el propósito de formación integral lo que apunta en el mismo sentido del informe sobre educación y género de la UNESCO (2017) señala que el acceso a la educación para niñas y mujeres ha mejorado a nivel mundial, sin embargo, existen todavía disparidades en el mundo.

\section{Los estudiantes de Contaduría Pública bajo el código de conducta profesional}

Durante el proceso análisis de la planeación didáctica, se identifica que los docentes establecen actividades colaborativas, trabajo en equipo y toma de decisiones, todas relacionadas con la convivencia y la forma en que interactúa el estudiante de la licenciatura de Contador Público. Las actividades incluyen un conjunto específico de tareas, que representan matices de un ambiente de aprendizaje donde se amplían y consolidan 
lazos de compañerismo y amistad, ejercitando la aceptación, compresión y compromiso de participación. Se identificaron actividades que parecían lecciones de convivencia en el salón de clases pero que trasladadas al ejercicio de la profesión ayudan a los estudiantes al reconocimiento y la definición de las situaciones de convivencia que tienen un impacto o que preocupan a la comunidad, por ejemplo, en las unidades de aprendizaje seminario de investigación y dirección estratégica se identifican los efectos en la economía de la informalidad de las empresas y su limitado crecimiento por no generar facturas. Se interpreta el diseño de estas actividades como respuesta a lo que ellos creen que son las prácticas de la empresa e involucran al estudiante, en un contexto donde la participación es limitada debido a la centralización de la toma de decisiones que tienen los empresarios. Uno de los docentes establece como aprendizajes actitudinales transversales en las actividades los valores de honestidad, honradez, veracidad e imparcialidad son ejercitadas por los estudiantes en la actividad. E incluso, aunque el estudiante revisa el código de conducta en las unidades de aprendizaje de segundo nivel, la unidad de aprendizaje de Seminario de Investigación hace hincapié en esto, cuando se trata de plantear un problema que resuelve desde su práctica profesional. Otro docente utilizó el debate como pregunta detonante del debate ¿cómo desde el ejercicio profesional del contador público se contribuye en la construcción de relaciones desde la sociedad con la empresa informal, a partir de su propio código ética profesional? desde la premisa de que los negocios informales tienen un efecto positivo en la economía al ser generadores de empleo.

El código ético profesional, representa lo que colectivamente los estudiantes creen que es moralmente correcto y las consecuencias prácticas de violar el código. Desde la perspectiva de los docentes y las estrategias emergentes en el desarrollo de la convivencia, en algunas actividades, está entretejida la perspectiva de género. Sin embargo, éstos no dominan en las secuencias didácticas que planean los docentes de las unidades de aprendizaje. Hasta el momento, se infiere que muchos de los docentes no refuerzan la convivencia desde la equidad de género.

\section{Interacción con los demás: contexto, participación y toma de decisiones}

Los estudiantes, tienen de forma activa los mecanismos institucionales, incluyendo contexto, participación y toma de decisiones -todas las diversas funciones se cumplen-. En concreto, estos mecanismos de influencia se extienden al modelado de la convivencia que fortalece la participación a través de las estrategias de aprendizaje, reglas institucionalizadas, y eventos académicos. Otras influencias son provocadas por las competencias de aprendizaje de la unidad de aprendizaje y el docente (por ejemplo, una convivencia con la interacción entre compañeros). A efecto de presentar las perspectivas de los docentes se definen los tres términos de la siguiente manera: (1) contexto: especifica aspectos inherentes a las condiciones y circunstancias de convivencia que se atribuyen a las actividades; (2) participación: crea ambientes que consolidad la aceptación; y (3) toma de decisiones: esos actos o medidas tomadas por los estudiantes relacionados con el contexto de democracia.

En el ambiente de aprendizaje, un docente diseña actividades colaborativas integradas al proceso de toma de decisiones como mecanismo que refuerza el comportamiento para aprender a convivir. Otro docente emplea para ampliar los conocimientos en las actividades con un grupo de estudiantes y pedir consejo a los miembros de grupo bajo el principio de compartir los mismos valores. Es decir, los estudiantes se encuentran en un continuo entre el cumplimiento forzoso y la internalización de las normas de convivencia, que tienen poco impacto en su toma de decisiones. En el otro extremo, los estudiantes integran sus creencias y prácticas, con su toma de decisiones. Mientras que muchos docentes en su secuencia didáctica plantean las actividades que generan y refuerzan significado del comportamiento de los estudiantes y formalizan y se explicitan, las normas de convivencia, políticas en el manejo del conflicto que están completadas en el programa de estudios de la unidad de aprendizaje en lo que ellos denominan encuadre de clase.

En el análisis de las planeaciones didácticas, se hizo evidente que el contexto promueve interacciones, con la intervención del docente, para que los estudiantes tomen acciones concretas con un comportamiento democrático. Estos y otras actividades revelaron que se definen intervenciones relacionadas con la convivencia para fomentar el comportamiento democrático de los estudiantes, por ejemplo, usan rúbricas de coevaluación. Aparte de simplemente proporcionar los saberes de convivencia del estudiante, estos elementos están incrustados dentro de comillas. En la secuencia, se incluyen actividades que estructuran las intervenciones de los estudiantes con implicaciones en las relaciones e interacciones de los estudiantes. Los docentes atribuyen muchos aspectos institucionales a mejores decisiones convivencia. Un docente describió, "proporcionar a los estudiantes un entorno de aprendizaje donde pueden llegar a conocerse no sólo como estudiante sino como persona". Otro docente hace referencia a que las clases pequeñas "permiten una comunicación más amplia y analizar la situación de mujeres y hombres, haciendo énfasis en la participación, responsabilidad democrática, pluralidad, identidad y valoración de las diferencias y calidad de las relaciones en contexto del aula o el ejercicio de la profesión." 


\section{Interacción con los demás para crear un sentido de convivencia}

Los docentes crean un sentido de convivencia, un sentido de compañerismo y amistad ejercitando la aceptación, compresión de los demás. Para este estudio, la convivencia puede ser formada por tres entradas. En primer lugar, el sentido de convivencia en los estudiantes vincula el código de ética profesional con el dialogo abierto y ponen en práctica los valores inherentes de los derechos humanos y la dignidad del otro y de sí mismo. En segundo lugar, se detecta que los temas de convivencia se fortalecen dentro del aula. Los docentes tienen en alta estima su contribución directa al desarrollo de la convivencia. Así que los docentes van más allá de simplemente señalar actividades de convivencia; hacen hincapié en la participación de las mayorías para tomar decisiones. Algunos atribuyen directamente al comportamiento tolerante, respetuoso, participativo y de cooperación como parte un todo y no sólo se centra en el aprendizaje dentro del aula, sino también fuera de la clase.

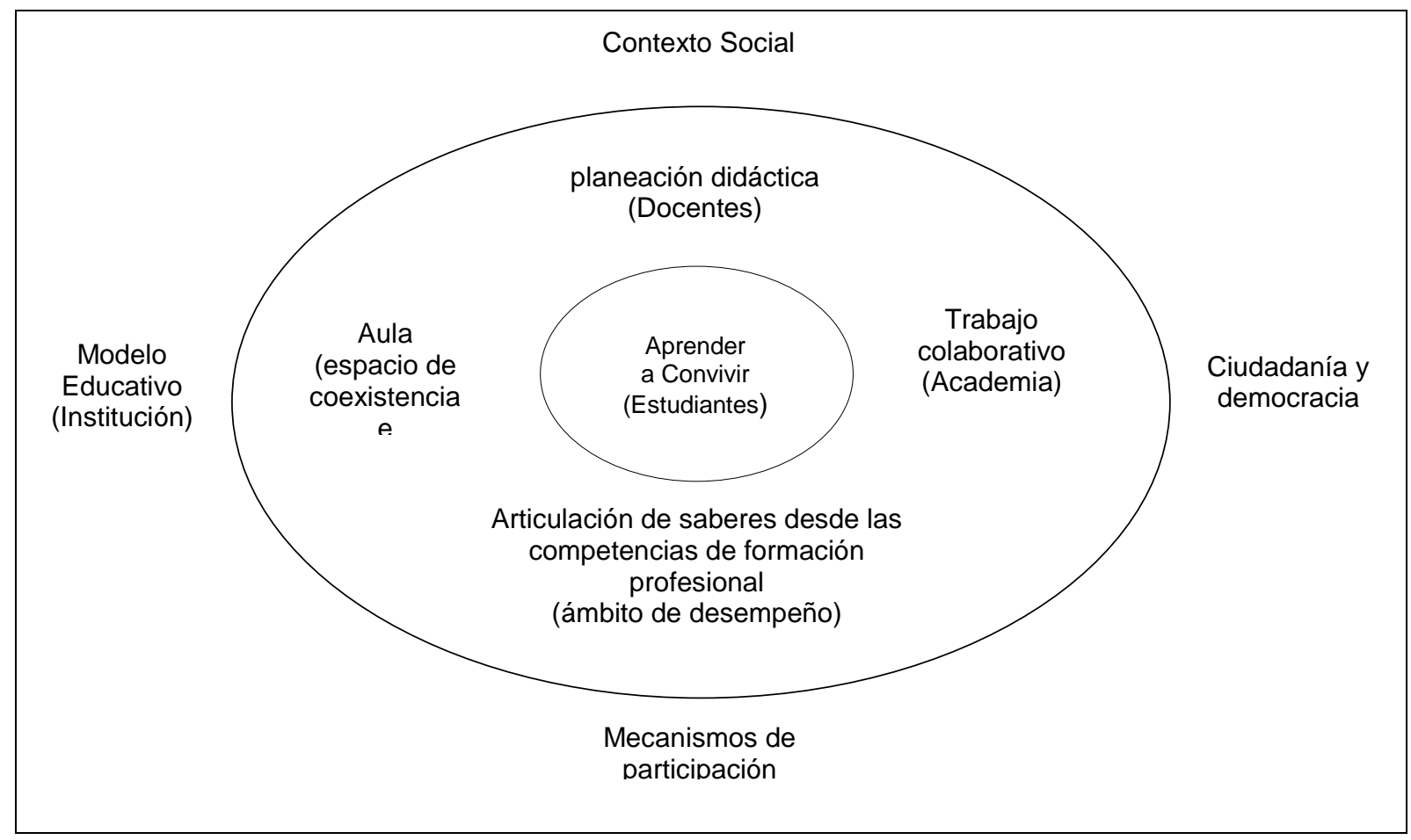

Fig. 1: Modelo de planeación didáctica que integra el saber convivir.

De los resultados del estudio de las planeaciones didácticas res se desprende el modelo de planeación didáctica que activa los saberes de la convivencia en las instituciones de la educación superior, véase figura 1. Los docentes que activan los saberes de convivencia en la sociedad democrática del siglo XXI diseñan una planeación didáctica argumentada desde una visión que subraya las diferencias individuales, las habilidades sociales y el deseo de participar en el planeamiento y la dirección del propio aprendizaje de los estudiantes, con lo que conceden gran importancia a la convivencia, a la individualidad, a la colectividad, a los ambientes de aprendizaje y la formación democrática. Las propuestas pedagógicas de los docentes están orientadas a una formación integral del estudiante y su contribución al desarrollo de la sociedad. Las instituciones de educación superior no solo deben formar para el ejercicio de la profesión en términos de una actuación libre sino también en términos de ciudadanía.

En el diseño de una planeación didáctica se establece la secuencia didáctica que comprende la intención de coordinar una serie de actividades de los estudiantes, para lo cual los docentes diseñan el objeto de aprendizaje con la planeación y programación de los diferentes recursos didácticos y materiales que se utilizarán en el desarrollo de ambientes de aprendizaje significativo. Una vez diseñada la secuencia se establecen las técnicas de aprendizaje en función de los estudiantes y se iniciará el trabajo según lo planeado para finalmente comprobará los desempeños de las y los estudiantes.

\section{LIMITACIONES E IMPLICACIONES}

Una constante en la investigación, derivada del procedimiento cualitativo en la recolección de información en las seis planeaciones didácticas, se extrae los datos de aprender a convivir y las estrategias didácticas de los docentes, se le atribuye un papel significativo en la construcción del significado que da el docente a la convivencia y la construcción de la democracia. El problema más revelador que se ha descubierto en el 
estudio es que el personal docente que trabaja en las aulas de las instituciones de educación superior, tiene la percepción de fomentar la convivencia y vincularla a contextos reales de la práctica profesional, con el diseño de ambientes de aprendizaje, como una aproximación con su realidad, con la intención de fortalecer la capacidad de participar en la resolución de los problemas de la sociedad. Desde esta arista de la investigación, se presenta el grave problema que giran alrededor del comportamiento de los estudiantes, pero no se incorporar al estudio la percepción de ellos, por lo que está incompleto ni tampoco se realizaron entrevistas a los docentes, así como no existe evidencia de que en los instrumentos de evaluación diagnóstica que el docente utilizó se consideren las actitudes con respecto a la convivencia.

\section{CONCLUSIONES}

Del análisis de los resultados mostrados y su reflexión con base en la revisión de la literatura se concluye que:

1) en las instituciones de educación superior se integra el aprender a convivir en el proceso de aprendizaje como parte de la formación integral de los egresados, se articulan saberes a partir de las estrategias didácticas que emplean los docentes, que sirven para definir y reconocer situaciones que limitan mayores niveles de responsabilidad, pluralidad, valoración de las diferencias y calidad de las relaciones en el aula o el ejercicio de la profesión y contribuyen a la construcción de una ciudadanía en los estudiantes que le da sentido a su actuación.

2) en el marco del modelo educativo, los docentes del programa de formación profesional de Contador Público, activan los saberes de convivencia orientados por el trabajo colegiado de las academias, destaca el énfasis en las diferencias individuales, habilidades sociales y formulación de actividades que promueven los valores éticos en el ejercicio profesional y de ciudadanía.

3) de la planeación didáctica, se identifica que se promueven la integración de saberes comportamentales, el sentido que los estudiantes asignan al código de ética profesional, con un dialogo abierto y puesta en práctica de los valores inherentes a los derechos humanos, la dignidad del otro y de sí mismo; y desde el sentido que el docente brinda a la formulación y ejecución de una propuesta de solución a los problemas de las empresas desde un enfoque contable como el entorno del ámbito de desempeño del futuro egresado.

\section{REFERENCIAS}

Bandura, A. y F. Menlove, Factors Determining Vicarious Extinction of Avoidance Behavior through Symbolic Modeling, doi: 10.1037/h0025260, Journal of Personality and Social Psychology, 8(2, Pt.1), 99-108 (1968)

Bicocca, M., El Camino del Conocimiento, Retos de la Educación Superior según Antonio Millán-Puelles, Estudios sobre Educación, ISSN: 1578-7001, 26, 219-234 (2014)

Castro-Alfaro, A., G. Marrugo-Peralta, J. Gutierrez-Hurtado e Y. Camacho-Contreras, La convivencia y la Mediación de Conflictos como Estrategia Pedagógica en la Vida Escolar, Revista Panorama Económico, ISSN: 0122-8900, (22), 169$190(2014)$

Celkan, G., L. Green y K. Hussain, Student Perceptions of Teacher Respect Toward College Students, doi: 10.1016/j.sbspro.2015.04.435, Procedia-Social and Behavioral Sciences, 191, 2174-2178 (2015)

Coll, C., E. Martín, T. Mauri y otros cinco autores, El constructivismo en el Aula, 9ª Ed., Graó, Barcelona, España (1999)

Cuesta, M. y A.K. Witt, How Gender Conscious Pedagogy in Higher Education can Stimulate Actions of Social Justice in Society, Journal of Social Inclusion, ISSN: 1836-8808, 2(1), 12-23 (2014)

Díaz-Barriga, A., El Docente y los Programas Escolares: Lo institucional y lo Didáctico, Universidad Nacional Autónoma de México, Instituto de Investigaciones sobre la Universidad y la Educación, México (2009)

Díaz-Barriga, A. y G. Hernández, Estrategias docentes para un aprendizaje significativo. Una interpretación constructivista, 2ª Ed., Mc Graw Hill, México (2002)

Domínguez, Y., J. Gutiérrez-Barroso y N. Gómez-Galdona, Equidad, Género y Diversidad en Educación, doi.org/10.19044, European Scientific Journal, 13(7), 300-319 (2017)

Fernández, I., Los Programas de Ayuda para la Mejora de la Convivencia en Instituciones Educativas, Bordón, Revista de Pedagogía, ISSN: 0210-5934, 60(4), 137-150 (2008)

Ferrer, A., Evaluación y Cambio de los Sistemas Educativos: La Interacción que Hace Falta, doi: http://dx.doi.org/10.1590/S0104, Ensaio: avaliação e Políticas Públicas em Educação,16(59), 275-296 (2008)

Fierro-Evans, C., G. Tapia y otros cuatro autores, Conversando sobre la Convivencia en la Escuela: Una Guía para el Auto-diagnóstico de la Convivencia Escolar desde las Perspectivas Docentes, Revista Iberoamericana de Evaluación Educativa, ISSN: 1989-0397, 6 (2), 103-124 (2013) 
Flores Pérez, J. y M. Lavín, Estudio Monográfico acerca de las Políticas de la Educación Superior Chilena en el Contexto de la Inclusión, Tesis Doctoral, Universidad Academia de Humanismo Cristiano, Santiago, Chile (2017)

García-Raga, L., R. Grau y R. López-Martín, Mediation as a Process for the Management of Conflict and the Improvement of Coexistence in Educational Centres. A Study Based on the Perceptions of Secondary School Students, doi: 10.1016/j.sbspro.2017.02.091, Procedia-Social and Behavioral Sciences, 237, 465-470 (2017)

Glaser, B.G., A. Strauss, Discovery of Grounded Theory: Strategies for Qualitative Research, 2ª Ed., Routledge, New York, USA (2017)

Gutiérrez-Méndez, D. y E. Pérez-Archundia, Estrategias para Generar la Convivencia Escolar, Ra Ximhai, ISSN: 16650441, 11(1), 63-81 (2015)

Gutmann, A., La Educación Democrática: Una Teoría Política de la Educación, Paidós Ibérica, Barcelona, España (2001)

Hess, D.J. y B., Collins, Climate Change and Higher Education: Assessing Factors That Affect Curriculum Requirements, doi: 10.1016/j.jclepro.2017.09.215, Journal of Cleaner Production, 170, 1451-1458 (2018)

Horbath, J.E. y M. Gracia, The Right to Education: An Analysis from the Recent Education Policy in Mexico in the Last Two Decades, doi:http://dx.doi.org/10.18359/ries.1373, Revista de Relaciones Internacionales, Estrategia y Seguridad, 11(1), 171-191 (2016)

lanni, N., La Convivencia Escolar: Una Tarea Necesaria, Posible y Compleja, Ciudadanía, Democracia y Valores en Sociedades Plurales, ISSN: 1728-0001, 2, 1-11 (2003)

Jares, X.R., Aprender a Convivir, Rev. Interuniversitaria de Formación del Profesorado, ISSN: 0213-8646, (44), 79-92 (2002)

Kim, J.S., Development of A Global Lifelong Learning Index for Future Education, doi: https://doi.org/10.1007/s12564-0169445-6, Asia Pacific Education Review, 17(3), 439-463 (2016)

Knowles, R. y Ch. Clark, How Common is the Common Good? Moving Beyond Idealistic Notions of Deliberative Democracy In Education, doi: 10.1016/j.tate.2017.12.002, Teaching and Teacher Education, 71, 12-23 (2018)

Martínez, M. y E. Prats, Citizenship Education in Spain in the Twenty-first Century, Citizenship Education around the World, Local Contexts and Global Possibilities, por J. Petrovic and A. Kuntz, 87-109, Nueva York, USA (2014)

Martínez, M., Educación y Ciudadanía en Sociedades Democráticas: Hacia una Ciudadanía Colaborativa, en Educación, Valores y Ciudadanía por Toro, M. y Tallone, A., OEI - Fundación SM, 59-71, Madrid, España (2011)

Ministerio de Educación Nacional de Colombia, Guía Pedagógica para la Convivencia Escolar, Guía N 49, Gobierno de Colombia (2013)

Nussbaum, M., Sin Fines de Lucro. Por Qué la Democracia Necesita de las Humanidades, Katz Editores, Madrid (2010)

Palacios, A., G. Mosquera, M., J. Rosero y R. Mosquera, La investigación como Estrategia para el Mejoramiento de la Convivencia Escolar y la Construcción de Ciudadanía, doi:https://doi.org/10.22395/csye.v5n10a4, Ciencias Sociales y Educación, 5(10), 101-115 (2016)

Pérez-Jorge, D., Y. Márquez-Domínguez y otros tres autores, Values Education as a Tool for Social Change from an Educational Approach, International Review of Social Sciences and Humanities, ISSN: 2248-9010, 11(1), 91-100 (2016)

Riviére, A., El sujeto de la Psicología Cognitiva, Alianza, Madrid, España (1987)

Torres-Rivera, A., M. Badillo, V. Kajatt y E. Ramírez, Las Competencias Docentes: el Desafío de la Educación Superior, Revista Innovación educativa, ISSN: 1665-2673, 14 (66), 129-145 (2014)

UNESCO, Cracking the Code: Girls' and Women's Education In Science, Technology, Engineering and Mathematics (STEM), París, Francia (2017)

UNESCO, La Educación Inclusiva: el Camino Hacia el Futuro. Documento de Referencia Cuadragésima Octava Reunión, Conferencia Internacional de Educación, 25 al 28 de noviembre, Ginebra, Suiza (2008)

UNESCO, Marco de Acción Dakar. Educación para Todos: Cumplir Nuestros Compromisos Comunes. Foro Mundial sobre Educación Dakar Senegal, París, Francia (2000)

Vélez, S., M. Lorenzo y J. Garrido, Leadership: its Importance in the Management of School Coexistence, doi: 10.1016/j.sbspro.2017.02.059, Procedia-Social and Behavioral Sciences, 237, 169-174 (2017)

Vygotsky, L.S., El desarrollo de los Procesos Psicológicos Superiores, Grijalbo, Barcelona, España (1979) 
